\title{
Congenital pericardial defect presenting as chest pain
}

\author{
R A Rusk, A Kenny
}

Congenital pericardial defects are rare, occurring in approximately 1 in 14000 necropsies. They are usually undetected during life and fewer than 200 cases are reported in world literature. ${ }^{1}$ Modes of presentation include chest pain, syncope, embolisation from mural thrombus, pericarditis or sudden death. We report a 34 year old woman with chest pain in whom the diagnosis was suggested by chest radiography and confirmed by magnetic resonance

\section{Department of Cardiology, Freeman Hospital, Freeman Road, Newcastle upon Tyne NE7 7DN, UK R A Rusk A Kenny \\ Correspondence to: Dr Rusk. \\ Accepted for publication 7 September 1998}

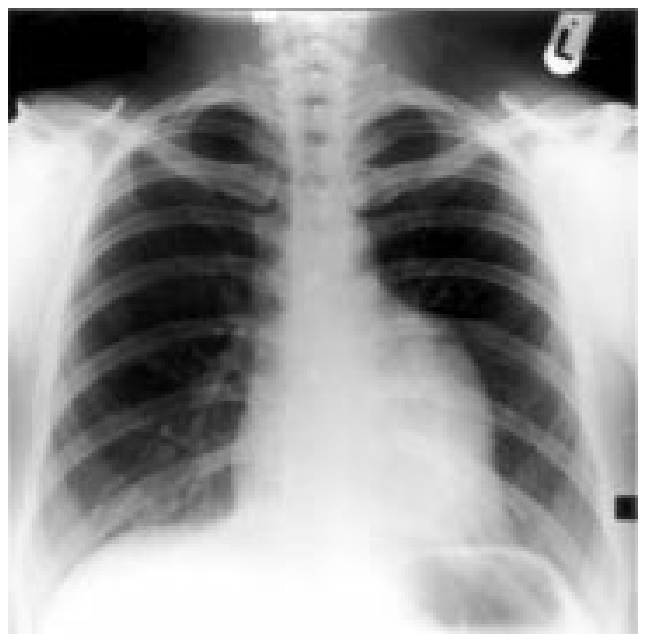

Figure 1 Postero-anterior chest $x$ ray showing convex prominence of the left heart border.

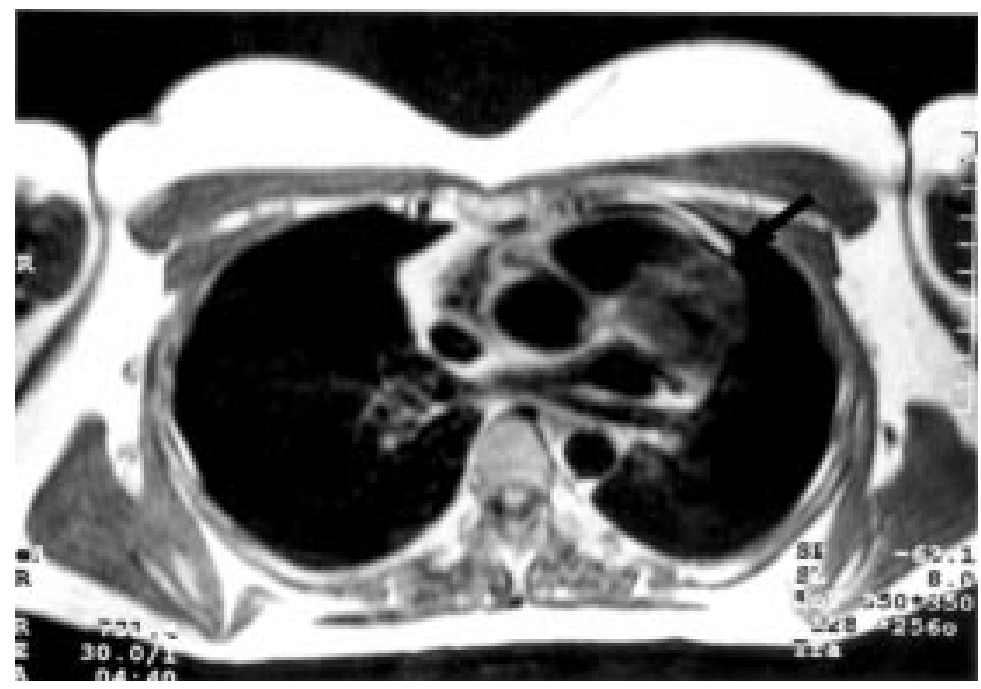

Figure 2 Magnetic resonance image showing part of the pericardial edge (arrow) bordering the defect. imaging. Management was by video assisted thoracoscopic partial pericardiectomy.

\section{Case report}

A 34 year old female cattle dealer developed central, non-pleuritic chest tightness radiating through to her back while standing. The pain eased spontaneously after 10 minutes but recurred overnight, waking her and easing only when she turned on to her right side. She had previously been healthy, she had had three normal pregnancies, and her work as a cattle dealer demanded a high level of physical fitness. On admission the pain settled spontaneously, and clinical examination and ECG were normal even during discomfort. Chest radiography (fig 1) showed a convex prominence of the left heart border. Chest computed tomography and transthoracic echocardiography were normal. Transoesophageal echocardiography showed diastolic bulging of part of the basal aspect of the left ventricle, which was also seen on ventriculography. Coronary arteriography was normal. The diagnosis was confirmed by magnetic resonance imaging (fig 2), which showed part of the left atrium and left ventricle protruding through a defect in the pericardium.

The pericardium was examined under general anaesthesia using video assisted thoracoscopy. A $3 \times 3 \mathrm{~cm}$ pericardial defect was seen overlying the left atrial appendage through which adhesions, branches of the circumflex artery, and the appendage could be seen. The defect was enlarged to create a $10 \times 10 \mathrm{~cm}$ window. The patient remains asymptomatic six months after the procedure.

\section{Discussion}

The favoured explanation of pericardial defects, which occur three times more commonly in men than women, is the persistence of a pleuropericardial foramen owing to arrested development of the pleuropericardial membrane. ${ }^{2}$ This may be caused by a deficient blood supply, and it has been hypothesised that the predominance of left sided defects results from premature atrophy of the left duct of Cuvier. The right duct of Cuvier normally persists as the superior vena cava thereby ensuring closure of the right pleuropericardial membrane.

Chest pain can be produced by one of several mechanisms including herniation of the left 
atrial appendage, tension of pleuralmyocardial adhesions formed in the absence of pleural pericardium, or mechanical infringement of the coronary vessels by a thickened edge of the remaining pericardium. ${ }^{2}$ The last of these is unlikely in our case as there were no ECG changes or evidence of migratory obstruction to coronary artery flow at arteriography. Previous reports confirm the positional nature of the pain, namely precipitation by lying on the left side and relief by turning to the right. $^{3}$

There is no consistently successful diagnostic technique. Chest radiography often raises suspicion, and in the past the diagnosis was frequently established by the presence of a pneumopericardium on induction of an iatrogenic left pneumothorax. ${ }^{4}$ Echocardiography and, more often, computed tomography or magnetic resonance imaging are now used to confirm the diagnosis, but their success is variable and sometimes surgical exploration is required. Surgical intervention, either by repair of the defect or by enlargement to relieve obstruction, is indicated in all recognised pericardial defects, not only to relieve symptoms but also because of the risk of sudden death. Death is not solely attributable to herniation of the left ventricle but may also occur with herniation of the left atrial appendage and involvement of the left circumflex coronary artery. $^{5}$

1 Risher WH, Rees AP, Ochsner JL, et al. Thoracoscopic resection of pericardium for symptomatic congenital resection of pericardium for symptomatic conge
pericardial defect. Ann Thorac Surg 1993;56:1390-1.

2 Wallace HW, Shen D, Baum S, et al. Angina pectoris associated with a pericardial defect. $\mathcal{F}$ Thorac Cardiovasc Surg 1971;61:461-5

3 Kostiainen S, Maamies TJ. Congenital partial absence of the left pericardium. Ann Chir Gynaecol 1975;64:40-3.

4 Morgan JR, Rodgers AK, Forker AD. Congenital absence of the left pericardium. Clincal findings. Ann Intern Med the left pericardiy

5 Robin E, Ganguly SN, Fowler MS. Strangulation of the left atrial appendage through a congenital partial pericardial defect. Chest 1975;67:354-5. 